\title{
Pantoprazole: An Unusual Suspect in a Patient with Fever
}

\author{
$\underline{\text { Nuno Melo }}^{1}$, Sílvia Policarpo ${ }^{2}$, Manuela Dias $^{1}$, Jorge Almeida $^{1}$ \\ ${ }^{1}$ Internal Medicine Department, Centro Hospitalar e Universitário de São João, Porto, Portugal \\ ${ }^{2}$ Infectious Disease Department, Centro Hospitalar e Universitário de São João, Porto, Portugal
}

Received: 06/05/2021

Accepted: 07/05/2021

Published: $28 / 05 / 2021$

How to cite this article: Melo N, Policarpo S, Dias M, Almeida J. Pantoprazole: an unusual suspect in a patient with fever. EJCRIM 2021;8: doi:10.12890/2021_002571..

Conflicts of Interests: The authors disclose no conflicts of interest.

Acknowledgements: We thank Professor Fernando Friões for reviewing the manuscript.

This article is licensed under a Commons Attribution Non-Commercial 4.0 License

\section{ABSTRACT}

Drugs can cause fever of unknown origin. Drug fever is a diagnosis of exclusion and can lead to unnecessary investigations and prolonged hospitalization. Any drug can be responsible. Here, we describe the case of a woman admitted because of acute hepatitis. Pantoprazole was started for stress ulcer prophylaxis when she was admitted to the ICU. Fever developed a few days later and an extensive diagnostic workup was negative. Fever remitted after pantoprazole discontinuation and the diagnosis of drug fever was established.

\section{LEARNING POINTS}

- Despite extensive diagnostic work-up, the aetiology of acute liver failure remains unclear in a large proportion of cases.

- Drug fever is a diagnosis of exclusion and must be considered in every patient with unexplained fever; any drug should be seen as a possible offending agent.

- Pantoprazole, a commonly prescribed drug, can be a rare cause of fever.

\section{KEYWORDS}

Drug fever, acute hepatitis, pantoprazole

\section{INTRODUCTION}

Drugs can cause of fever of unknown origin. It is a diagnosis of exclusion and is confirmed when fever resolves after suspension of the drug in the absence of another cause. The most frequently associated drugs are antibiotics, anticonvulsants and antiarrhythmic agents ${ }^{[1]}$.

\section{CASE DESCRIPTION}

A 42-year-old woman with a previous history of well-controlled asthma, alcoholism and breast cancer was admitted to the emergency department due to malaise and anorexia with 1 week of evolution. On admission, she was febrile $\left(38.3^{\circ} \mathrm{C}\right)$, blood pressure was $103 / 76$ $\mathrm{mmHg}$, heart rate was $110 \mathrm{bpm}$, and she presented with mild icterus and painful hepatomegaly. Blood analysis showed acute hepatitis (AST: 428 IU/I, ALT: 205 IU/I, GGT/AP: 1884/426 IU/I) with hyperbilirubinaemia (total bilirubin: 3.27 mg/dl and direct bilirubin: 1.79 mg/dl) with normal coagulation tests and slightly elevated C-reactive protein (CRP: $29.4 \mathrm{mg} / \mathrm{l}$ ). Viral serologies (HBV and HCV) were negative and abdominal ultrasonography only showed mild hepatomegaly with no additional pathological findings. She denied the use of recreational drugs or intentional drug poisoning. 
The patient was admitted to the general ward. On day 1 , she showed signs of grade 1 encephalopathy and her blood results showed a marked rise in transaminases (AST/ALT: 11937/1472 IU/I) with increased hyperbilirubinaemia (total bilirubin: $6.8 \mathrm{mg} / \mathrm{dl}$ and direct bilirubin: 3.91 $\mathrm{mg} / \mathrm{dl}$ ), de novo prothrombin time prolongation (PT: $22.2 \mathrm{sec}$, INR: 2.2) and low fibrinogen (106 mg/dl). Acute liver failure was diagnosed and the patient was transferred to an intensive care unit. Pantoprazole for stress ulcer prophylaxis and N-acetylcysteine (NAC) (initial loading dose of $150 \mathrm{mg} / \mathrm{kg} / \mathrm{hour}$ of NAC over 1 hour, followed by $12.5 \mathrm{mg} / \mathrm{kg} / \mathrm{hour}$ for 4 hours, then continuous infusions of $6.25 \mathrm{mg} / \mathrm{kg}$ for the remaining 67 hours) for putative toxic hepatitis, were started. Abdominal Doppler ultrasonography excluded portal vein or inferior vena cava thrombosis, and a larger panel of viral serologies (including HSV, HIV, CMV, HAV, HEV and EBV) was negative. Acetaminophen was undetectable in plasma, and no substances of abuse were found in urine. A liver autoimmune panel was negative. Alpha- 1 antitrypsin was normal. Urinary copper and ceruloplasmin were both normal. Ferritin was elevated, but serum iron and transferrin were both normal.

By the following day, hepatic function had dramatically improved: coagulation tests were normal, there were significant drops in serum transaminases and total bilirubin, and the patient presented no signs of encephalopathy. Despite the clinical and analytical improvement, a liver biopsy was taken, which showed mild iron overload with no additional clues to a specific aetiology.

However, the patient developed nicotine withdrawal symptoms which precluded transfer to the general ward. She had one episode of lowgrade fever, which was interpreted as part of the withdrawal syndrome. She was transferred to the general ward after 6 days in the ICU. Liver function was normal and serum transaminases continued to decline. Despite the absence of any symptoms or signs of infection, the patient presented low-grade fever (approximately one episode per day, $\sim 38^{\circ} \mathrm{C}$ ) during the following days. Since she had no new symptoms and physical examination was unremarkable, antibiotics were withheld. Blood and urine cultures were negative. Renal function and urinalysis were normal. Chest and abdominopelvic CT scans were normal and a transthoracic echocardiogram showed no signs of infective endocarditis. C-reactive protein and the erythrocyte sedimentation rate were low. Several drugs were suspended but the fever persisted. Anti-nuclear antibodies, ANCA and anti-dsDNA were negative and a Doppler ultrasound of temporal arteries was normal. A PET-CT scan was normal. Eventually, pantoprazole was suspended and the febrile episodes resolved. A diagnosis of drug fever associated with pantoprazole was made and the patient was discharged. She remained well, without any new febrile episodes, during 1 year of follow-up.

\section{DISCUSSION}

The authors present a case report of a patient with acute hepatitis of uncertain aetiology complicated with drug fever associated with pantoprazole. Acute liver failure is a rare condition and a large proportion of cases are of unknown origin ${ }^{[2]}$. Only a few causes are associated with massive cytolysis with AST >10,000 IU/I, such as paracetamol or MDMA poisoning, acute hepatitis B virus infection or ischaemic hepatitis. Our patient denied intentional drug poisoning or use of recreational drugs and the diagnostic work-up was inconclusive. Despite the severity of liver illness, hyperacute presentations have a much greater chance of spontaneous recovery ${ }^{[2]}$, as documented in this case report.

Fever can be the sole manifestation of an adverse drug reaction in 3-5\% of cases. The true frequency of drug fever is unknown due to underreporting and misdiagnosis ${ }^{[1]}$. A wide range of drugs can cause drug fever, but the most commonly associated are antibiotics, anticonvulsants and antiarrhythmic agents ${ }^{[1]}$. To our knowledge, only one case of drug fever associated with pantoprazole has been reported ${ }^{[3]}$.

The key to making the diagnosis is to consider drugs as a possible cause of fever in any patient. Infection is the most common cause of fever and the most common clinician's response to a patient with fever is to search for infection, which may lead to antibiotic over-prescription, increasing the risk of side effects and the development of antimicrobial resistance when infection is absent ${ }^{[4]}$. Our patient was submitted to an extensive diagnostic work-up before the diagnosis of drug fever was considered, and the paucity of data about drug fever associated with pantoprazole led to further testing before the diagnosis was made.

Clinical features of drug fever vary with the offending drug, but some clues may aid in the diagnosis: patients with this condition often appear inappropriately well for the degree of fever that they have, and they are frequently unaware of the fever ${ }^{[1]}$. Another potential clue is the temporal relationship with drug administration: drug fever may present any time following the initiation of therapy, but it frequently occurs after 1-2 weeks of treatment. Our patient did not have any symptoms and fever appeared 6 days after the first pantoprazole administration. Although blood investigations can be helpful in the diagnosis of drug fever, results are highly variable and there is no single laboratory test for this condition. An elevation of the erythrocyte sedimentation rate (ESR) is sometimes found in drug fever and some patients may present eosinophilia ${ }^{[5]}$. In our case, blood tests were non-contributory for the diagnosis.

In conclusion, a high index of suspicion along with careful history making is required to diagnose drug fever, after the exclusion of infection and other more frequent aetiologies. Drug fever is a diagnosis of exclusion that must be considered early to avoid costly, inappropriate and potentially harmful investigations and therapies. 


\section{REFERENCES}

Patel RA, Gallagher JC. Drug fever. Pharmacotherapy 2010;30(1):57-69.

Bernal W, Auzinger G, Dhawan A, Wendon J. Acute liver failure. Lancet 2010;376(9736):190-201.

Schiller D, Maieron A, Schöfl R, Donnerer J. Drug fever due to a single dose of pantoprazole. Pharmacology 2014;94(1-2):78-79.

Harris LF, Holdsambeck HK. Drug fever--surprisingly common and costly. Ala Med 1986;56(3):19-22.

Cunha BA. Antibiotic side effects. Med Clin North Am 2001;85(1):149-185. 